\title{
Carillas directas de composite, para la rehabilitación estética de incisivos laterales rudimentarios: Reporte de caso
}

\author{
Direct composite veneers, for the aesthetic rehabilitation of rudimentary lateral \\ incisors: Case report
}

Valeria Alejandra Mendoza Larrea (b) ${ }^{1 a}$, María José Jarrìn Peñafiel (c) 1a

\section{RESUMEN}

El objetivo del presente caso es la descripción y evolución del tratamiento restaurador estético realizado con resinas compuestas, a órganos dentarios del sector anterosuperior con alteraciones de tamaño y forma. El presente caso trata de una paciente femenina de 15 años de edad, sin antecedentes médicos relevantes quien asistió a la clínica odontológica SERODU, por inconformidad estètica al momento de sonreír, al examen clínico se observó microdoncia en las piezas 12 y 22 con una forma de clavija. Se propuso como plan de tratamiento la restauración estética del sector anterosuperior con carillas en resina compuesta. A los 6 meses se observa clínicamente carillas en buen estado, sin ningún cambio en el color y ausencia de enfermedad periodontal.

Palabras clave: Composite; Anomalías Dentarias; Adolescente. (Fuente: DeCS BIREME)

\begin{abstract}
The objective of the present case is the description and evolution of the aesthetic restorative treatment carried out with composite resins, to dental organs of the anterosuperior sector with changes in size and shape. The present case deals with a 15-year-old female patient, without relevant medical history who attended the SERODU dental clinic, due to aesthetic disagreement at the time of smiling, the clinical examination observed microdontia in parts 12 and 22 with a form of plug. The aesthetic restoration of the anterosuperior area with composite resin veneers was proposed as a treatment plan. At 6 months, veneers are observed clinically in good condition, without any change in color and absence of periodontal disease.
\end{abstract}

Key words: Composite; Dental anomalies; Adolescent. (Source: MeSH NLM)

Recibido: 23 de setiembre de 2020

Aceptado: 20 de febrero de 2021

En línea: 08 de abril de 2021

${ }^{1}$ Universidad UTE. Ecuador

a Odontóloga

Este es un artículo de acceso abierto distribuido bajo la licencia Creative Commons Atribución 4.0 Internacional (CC BY 4.0) https://creativecommons.org/licenses/by/4.0/deed.es

\section{Correspondencia:}

Marì Josè Jarrìn

Dirección: Calle Bolivar 6-38 y Gonzàlez Suàrez

Correo electrónico: majp_1995@outlook.com

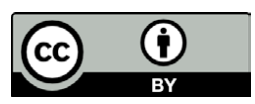

Citar como: Mendoza Larrea V, Jarrín Peñafiel MJ. Carillas directas de composite, para la rehabilitación estética de incisivos laterales rudimentarios: Reporte de caso. KIRU. 2021; 18(2):97-102. https://doi.org/10.24265/kiru.2021.v18n2.04 


\section{INTRODUCCIÒN}

El tipo de tratamiento de las anomalías dentarias se debe seleccionar teniendo en cuenta los requisitos funcionales y estéticos $(1,2)$. Existen varias posibilidades de tratamiento aceptables para devolver el tamaño y la forma a un órgano dentario. Dentro de estas, está la restauración de los órganos dentarios alterados con técnicas restaurativas directas con resina compuesta y la restauración con técnicas indirectas como carillas de cerámica, que desarrollan la morfología normal de los órganos dentarios $y$, a su vez, logran el cierre de diastemas $\left.{ }^{(3,4}\right)$. Las carillas directas con resinas compuestas se proponen como una técnica conservadora y rápida para la rehabilitación estética del sector anterior, ya que permite la aplicación del material de resina compuesta sin reducción del diente, donde el objetivo final es conservar la estructura dentaria natural sana y lograr la estética ${ }^{(5,6)}$.

Las carillas directas de composite presentan como beneficio bajo costo, amplia variedad de colores, fácil fabricación, reparación, y terminado, esencial para la rehabilitación estética del sector anterior ${ }^{(7,8)}$.

El objetivo del presente caso es la descripción y evolución del tratamiento restaurador estético realizado con resinas compuestas, a órganos dentarios del sector anterosuperior con alteraciones de tamaño y forma

\section{Presentación del caso}

Se presenta a la consulta una paciente de sexo femenino de 15 años de edad que refiere presentar disconformidad estética de sus dientes. No manifiesta antecedentes personales de importancia.

Al realizar el examen intraoral se observó microdoncia de las piezas dentarias 12 y 22 , los cuales presentaban forma de clavija. No se halló signos de enfermedad periodontal.

Se tomaron modelos de estudio que permitieron analizar mejor el caso.

Diagnóstico: Microdoncia localizada y dientes en forma de de las piezas dentarias 12 y 22.
Plan de tratamiento: Devolver la anatomía dental mediante el uso de carillas directas de composite.

\section{Ejecución - evolución del plan de tratamiento.}

El plan de tratamiento se inicia con los modelos de estudio y un encerado diagnóstico para planificar la forma, tamaño de los dientes así como y oclusión. (Figuras 1A, 1B Y 1C).

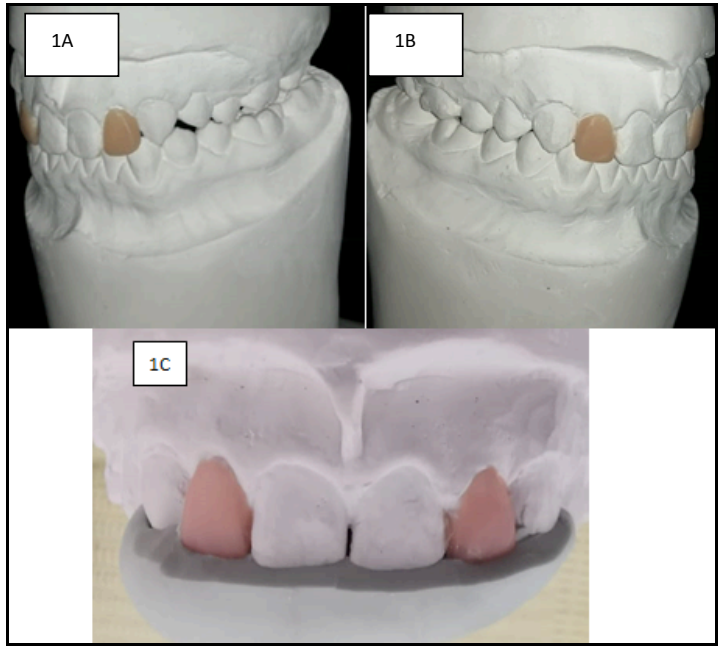

FIGURA 1 A, B y C. Vistas laterales y vista frontal de modelos de estudio con encerado de diaanóstico con cera Yeti”

Se seleccionó el color de la resina Opallis FGM con la que se realizó el tratamiento restaurador, se probó el color $A 1$ y $A 2$ de esmalte y $\mathrm{A} 1, \mathrm{~A} 2$ de dentina, colocando un incremento de resina sobre el esmalte de la pieza 21. Determinando que el color que armonizaba con las piezas dentarias contiguas era $\mathrm{A} 2$ de esmalte y $\mathrm{A} 1$ de dentina (Figura 2A). Se utilizó cepillo profiláctico en copa, piedra pómez de 45 micras y clorhexidina al $2 \%$, para realizar la remoción mecánica de placa dental (Figura 2B).

Se lavó y secó las piezas dentarias anteriores 12 y 22 por 5 segundos para realizar la desmineralización del esmalte dental con ácido ortofosfórico al $37 \%$ por 15 segundos con el fin de obtener micro porosidades y aumento de la energía superficial para mejorar la adhesión de la resina al órgano dental (Figura 2C). 
Se lavó por 30 segundos la superficie del esmalte de cada pieza dentaria para asegurarnos de eliminar restos de ácido ortofosfórico y prevenir sensibilidad post operatoria (Figura 2D). Posteriormente se colocó el adhesivo de quinta generación de la casa Ivoclar Vivadent "Tetric N- Bond Universal adhesive (Ivoclar- Vivadent, Schaan, Liechtenstein), aplicado aire con la jeringa triple por 5 segundos para eliminar el solvente del vehículo, fotocurado con luz halógena por 20 segundos (Figura 2E). Se colocó incrementos de $1 \mathrm{~mm}$ de resina microhíbrida OPALLIS FLOW (FGM, Brasil), correspondiente a dentina color A1, fotocurado con luz halógena por 40 segundos, incremento de la segunda capa de $1 \mathrm{~mm}$ de dentina A1 fotocurado por 40 segundos más, incrementos de esmalte tono A2 y fotocurado por 40 segundos siguiendo recomendaciones del fabricante (Figura 2F). El pulido se inició con el retiro de los excesos groseros con una fresa diamantada de grano fino para pulir resina y luego con copas, puntas y discos siliconados con el fin de alisar la superficie y evitar la acumulación futura de placa bacteriana, los discos de óxido de aluminio junto con las tiras de lija interdental ayudan a la reducción de excesos de las caras proximales mesial y distal, definiendo y delimitando la forma armoniosa de los incisivos laterales (Figura 2G). Para abrillantar la restauración ocupamos pasta para pulir resina, DIAMOND GLOSS a base de óxido de aluminio de grano extrafino con una fresa de felpa (Figura $2 \mathrm{H}$ ).

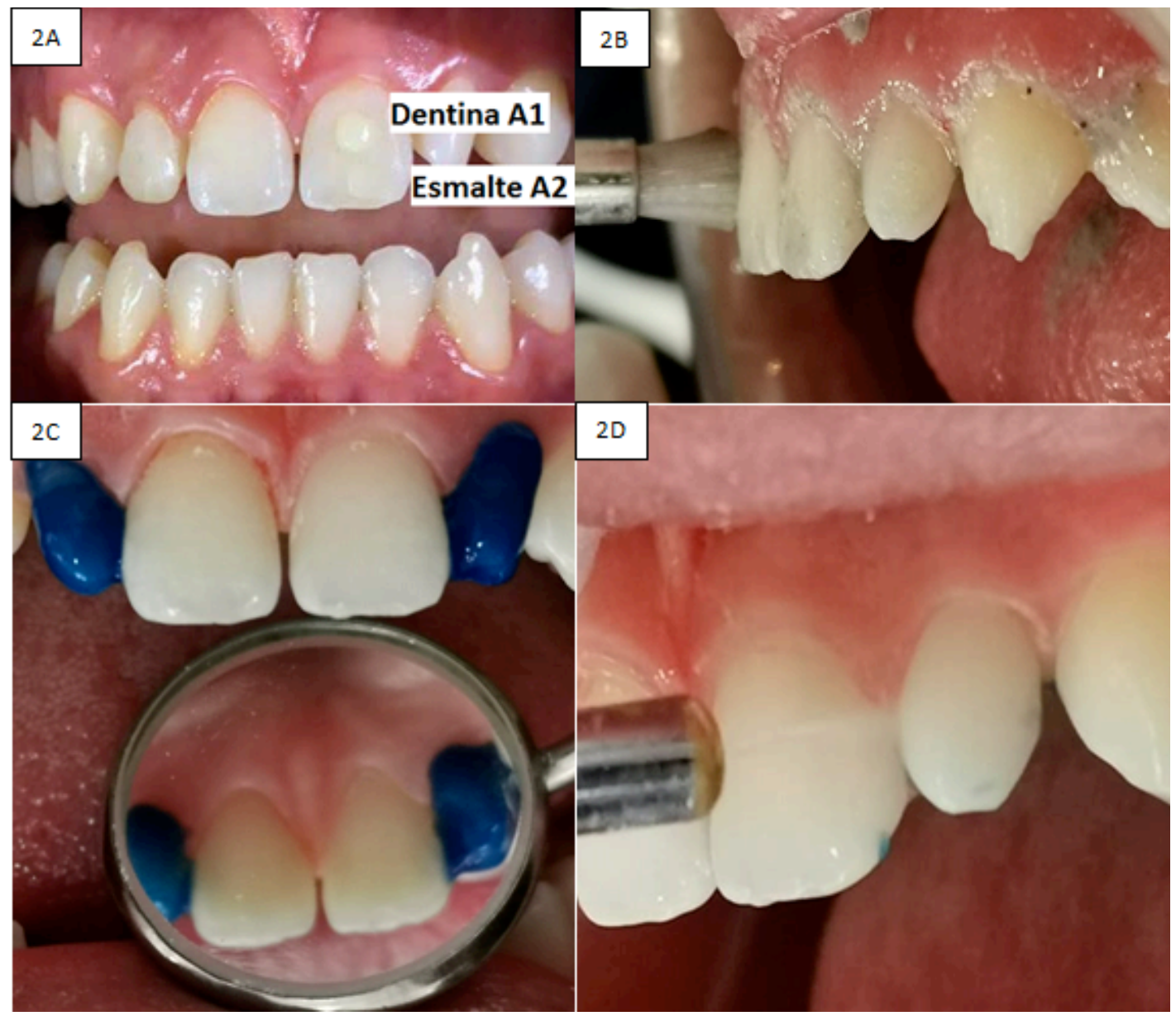

FIGURA 2.A. Elección de color de la resina para esmalte y dentina. B. Profilaxis dental a base de piedra pómez y clorhexidina. C. Grabado con ácido ortofosfórico al $37 \%$ por 15 segundos. D. Lavado por 30 seaundos de la superficie de óraanos dentales 12 v 22. 

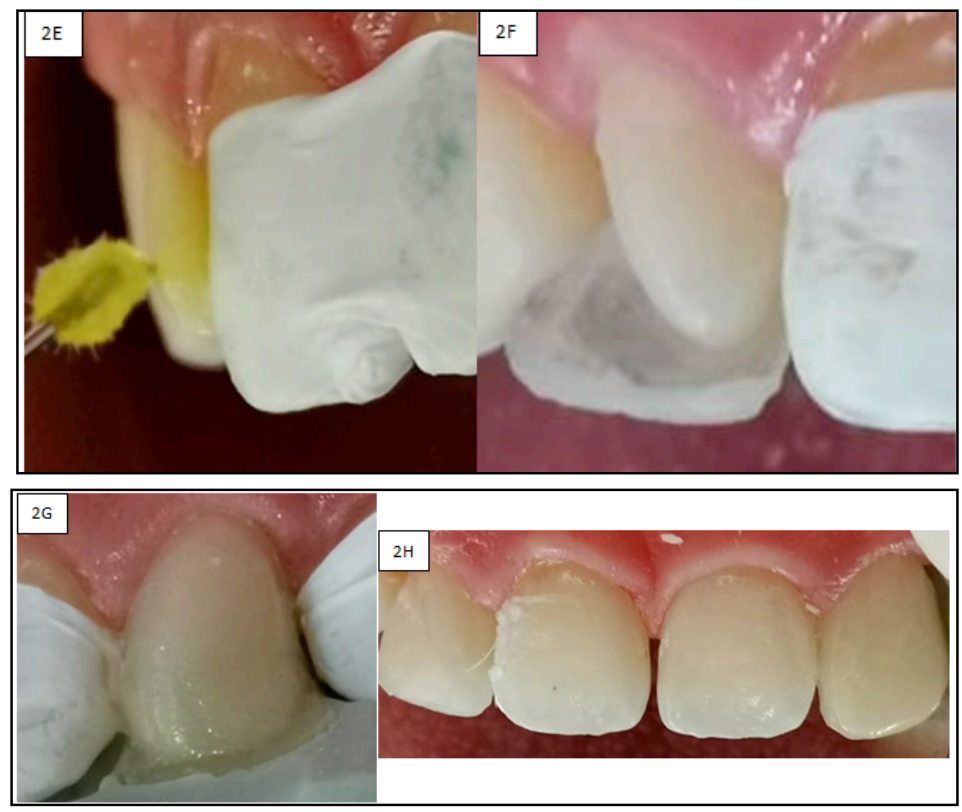

Figura 2E. Adhesivo de quinta generación Ivoclar Vivadent "Tetric N- Bond Universal colocado en la superficie de órganos dentales 12 y 22. 2F. Resina Opallis FGM de esmalte A2 colocada en la superficie palatina de órgano dental 12. 2G. Resina Opallis FGM correspondiente a dentina A1 colocada en el órgano dental 22. 2H. Resina Opallis FGM de esmalte A2 colocada en la superficie de órgano dental 12 y 22.

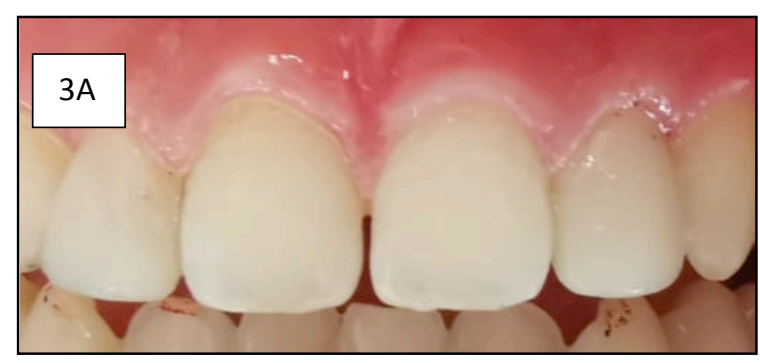

Figura 3A. Pulido final

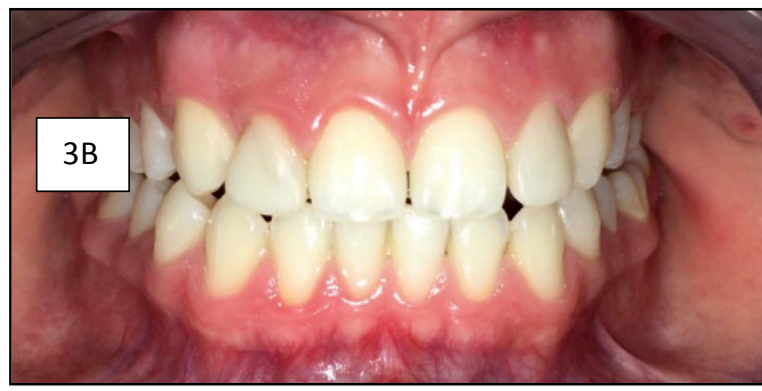

Figura 3B. Control clínico a los 6 meses
Se devolvió la armonía del sector anterior mediante el uso de una técnica mínimamente

invasiva en la que no se realizó ningún tipo de desgaste dental en las piezas 12 y 22.

Se redujo el espacio interproximal, y se facilitó el uso de la resina mediante la utilización de la llave pesada de silicona de condensación, ya que esta actuaba como molde o guía en base a nuestro encerado de diagnóstico (Figura 3A).

Seguimiento del tratamiento ejecutado. Se realizó control a los seis meses y en la exploración clínica se observaron carillas en buen estado y buena salud periodontal. El paciente no refirió sintomatología (Figura 3B).

\section{Discusión}

El procedimiento más utilizado es la restauración con carillas estéticas, cuyos materiales de elección son la cerámica en el caso de restauraciones indirectas y resinas compuestas en el caso de las restauraciones directas ${ }^{(9,10)}$. La cerámica conserva 
propiedades físicas y estéticas que la convierten en la primera opción; sin embargo, las resinas compuestas se convirtieron en una alternativa confiable $(11,12)$

KIRU.2021. Abr-Jun; 18(2): 97-101

Las técnicas de restauración directas y conservadoras con composite son una alternativa a las restauraciones en cerámica por la gran disponibilidad en el mercado de resinas con buena capacidad de pulido, dureza y resistencia al desgaste ${ }^{(13,14)}$. Estas restauraciones pueden ser modificadas $y$ pulirse in situ, lo que disminuye el tiempo de trabajo y costos ${ }^{(15,16)}$.

Las resinas compuestas son materiales resistentes, estéticos y durables que pueden emplearse en tratamientos estéticos ${ }^{(17)}$.

Se garantiza un tratamiento restaurador exitoso, siempre que se haya realizado un adecuado examen de la oclusión y función del paciente, se tome en cuenta la destreza del operador y la cooperación con los hábitos alimentarios del paciente ${ }^{(18)}$.

En un paciente muy joven es recomendable la utilización de carillas de composite ya que se considera a estas como un tratamiento reversible fácil de retirar y reparar, de manera que si el paciente en edad adulta desea colocarse una carilla de cerámica se sustituye de manera sencilla ${ }^{(19)}$. Incluso se elige este tipo de tratamiento en adolescentes, debido a que las carillas de cerámica involucran hacer un desgaste de la superficie del esmalte y este termina su etapa de maduración a los 18 años, por lo que es mejor esperar que pase este periodo y a fin de no dejar los dientes debilitados con predisposición a la caries dental ${ }^{(20)}$, es por estas razones que se eligió este tipo de tratamiento en el presente caso.

\section{CONCLUSIÒN}

Se obtuvo un resultado estético satisfactorio en el caso descrito, las carillas directas de composite mostraron ser una alternativa útil de bajo costo, fácil manipulación, con variedad de colores y texturizaciones que permitieron observar buenos resultados en un corto tiempo de trabajo.

Contribución de autoría: VML y MJJP participaron en el diagnóstico y tratamiento del caso clínico. Redactaron y aprobaron el manuscrito.

Fuente de financiamiento: autofinanciado
Carillas directas de composite, para la rehabilitación estética de incisivos laterales rudimentarios

Conflictos de interés: no hubo ningún conflicto de interés.

\section{REFERENCIAS BIBLIOGRÁFICAS}

1. Molina $B$, Montes de Oca Fernández $L$, Gamboa Mutuberría F. Embriología $Y$ Anatomía De La Cavidad Oral Y Faringe. Sociedad española de otorrinolaringología y patología CervicoFacial SEORL PCF. Libro virtual de formación en ORL. Alcalá de Henares. Madrid. SEORL PCF; 2014. p. 1-14

2. Albertí Vázquez, L, Más Sarabia, $M$, Martínez Padilla, S, Méndez Martínez, MJ. Histogénesis del esmalte dentario. Consideraciones generales. Archivo Médico de Camagüey [Internet]. 2007.[citado 20 Agosto 2019]; 11(3). Disponible en: http://www.redalyc.org/articulo.oa?id=211 118126015

3. Gresnigt MM, Kalk W, Ozcan M. Randomized controlled split-mouth clinical trialof direct laminate veneers with two micro-hybrid resin composites. J Dent. 2012;40(9):766-75.

4. Aliaga Muñoz B. La odontogénesis. Andalucía educa. [Internet]. 2017. [citado 20 Agosto 2018]; 201(9):53. Disponible en:

http://www.andaluciaeduca.com/hemerot eca/ae digital201.pdf\#page $=53$

5. Ceballos D, Espinal G, Jones M. Anomalías en el Desarrollo y Formación Dental: Odontodisplasia. Int. J. Odontostomat. [Internet]. 2015. [citado 20 Agosto 2019]; 9(1):129-136. Disponible en: https://scielo.conicyt.cl/pdf/ijodontos/v9n1 lart20.pdf

6. González $M$, Sánchez $D$, Delgado $T$, Castellanos C, Llamas C, López F, Segura E. Anomalías y displasias dentarias de origen genético-hereditario. Av. Odontoestomatol. [Internet]. 2012. [citado 20 Agosto 2019]; 28(6):287-301. Disponible http://scielo.isciii.es/pdf/odonto/v28n6/ori ginal3.pdf

7. Bedoya A, Collo L, Gordillo L, Yusti-A, Tamayo JA, Pérez A, Jaramillo $M$. Anomalías dentales en pacientes de ortodoncia de la ciudad de Cali, Colombia. Rev CES Odont. [Internet]. 2014. [citado 20 Agosto 2019]; 27(1):4554. Disponible en: 
http://www.scielo.org.co/pdf/ceso/v27n1/v 27n1a05.pdf

8. Carmona L. Diente cónico: presentación de dos casos. Rev. Méd. Risaralda. [Internet].2014.[citado $20 \quad$ Agosto KIRU.2021. Abr-Jun; 18(2): 97-102

9. D'La Torre Ochoa C, Gurrola B, Casasa A.Multidisciplinary approach in patient with upper lateral incisor microdontia. Case report. Revista Mexicana de Ortodoncia 2016; 4(2): 132-137.

10. Mandri MN, Aguirre A, Zamudio ME. Sistemas adhesivos en Odontología Restauradora. Odontoestomatología [Internet]. 2015.[cited 20 July 2019] ; 17( 26 ): 50-56. Available from: http://www.scielo.edu.uy/scielo.php?scrip $\mathrm{t}=$ sci_arttext\&pid=S168893392015000200006

11. Terry D, Barkmeier W, Ficher N, Nojiri K, Nagura $\mathrm{Y}$, Takamizawa $\mathrm{T}$, Latta $\mathrm{M}$, Miazaki M.Wear of resin composites: Current insights into underlying mechanisms, evaluation methods and influential factors. Japanese Dental ScienceReview. $\quad$ 54(2), 76-87. Recuperado de

https://www.ncbi.nlm.nih.gov/pmc/articles /PMC5944074/

12. Romero FM. Diastema. Una solución con una restauración provisoria de Cerómero. Revista Dental de Chile. 2005; 25: 20-25.

13. Hervás A, Martínez MA, Cabanes J, Barjau A, FosGalve P. Resinas compuestas: Revisión de los materiales e indicaciones clínicas. Med Oral Patol. [Internet]. 2006. [citado 17 Julio 2019]; 11(2):215-220. Disponible en: http://scielo.isciii.es/scielo.php?script=sci arttext\&pid=S1698-

69462006000200023\&lng=es.

14. Valerio I, Murillo LF. Reconstrucción de incisivos fracturados con resina nanohíbrida: reporte de un caso. Revista
2019];20(2):125-128.Disponible en: http://www.scielo.org.co/pdf/rmri/v20n2/v 20n2a10.pdf

Mendoza Larrea V. \& Jarrín Peñafiel MJ

Científica Odontológica.2010 [Internet]. 2010. [citado 17 Julio 2019]; 6(1). Disponible en: http://www.redalyc.org/articulo.oa?id=324 227911006

15. Gresnigt MM, Kalk W, Ozcan M.

Randomized clinical trial of indirect resin composite and ceramic veneers: up to 3year follow-up. J Adhes Dent. 2013;15(2):181-90.

16. Spyrides SMM, Bastian FL. In vitro comparative study of the mechanical behavior of a composite matrix reinforced by two types of fibers (polyethylene and glass). Mater SciEng C. 2014;24(5):6717

17. Meereis CTW, Leal FB, Lima GS, De Carvalho R V., Piva E, Ogliari FA. BAPO as an alternative photoinitiator for the radical polymerization of dental resins. Dent Mater. The Academy of Dental Materials; 2014;30(9):945-53.

18. Sarmiento $M$, Trujillo $C$, Mena $D$ y Mejìa K. Opacidad y traslucidez de diferentes resinas compuestas de acuerdo a su tamaño de partícula y su aplicación clínica.Acta Colombiana.2012; 2(1):101-11

19. Orozco J. Berrocal J, Diaz A. Carillas de composite como alternativa a carillas cerámicas en el tratamiento de anomalías dentarias. Reporte de un caso. Rev Clin Periodoncia Implantol Rehabil Oral. 2015; 8(1): 79-82.

20. Gomes G, Perdigão J. Prefabricated composite resin veneers-a clinical review.J Esthet Restor Dent. 2014; 26(5):302-13.

Valeria Mendoza Larrea

ORCID iD: 난 http://orcid.org/0000-0001-7986-1599

Valemend1994@hotmail.com

Jarrin Peñafiel JM

ORCID iD: (๑) http://orcid.org/0000-0002-1875-1254

majp_1995@outlook.com 
Copyright $\odot$ La revista. La revista Kiru es publicada por la Facultad de Odontología de la Universidad de San Martín de Porres, en Lima, Perú. 